\title{
DETERMINANTES DE LA DECISIÓN DE LOS MOTOTAXISTAS EN CARTAGENA (COLOMBIA) DE INFRINGIR LA MEDIDA DE PICO Y PLACA*
}

\author{
MARTHA YÁNEZ CONTRERAS*** \& KARINA ACEVEDO GONZÁLEZ*** \\ UNIVERSIDAD DE CARTAGENA
}

Recibido/ Received/ Recebido: 20/09/2013 - Aceptado/ Accepted / Aprovado: 19/03/2014

\begin{abstract}
Resumen
El mototaxismo es un tipo de transporte público informal que se ha tratado de controlar en la ciudad de Cartagena (Colombia) con regulaciones como la medida de pico y placa. No obstante, esta regulación registra una alta tasa de infracción (cerca del 69\% de los mototaxistas la infringen). En este documento se estiman modelos de elección binaria en los que se buscan examinar los factores que hacen más propenso a un mototaxista a infringir la regulación. Los resultados muestran que los determinantes más robustos son el número de motocicletas para trabajar y la ganancia esperada. Se concluye que la medida ha incentivado el uso de por lo menos una motocicleta adicional.

Palabras clave: Mototaxismo, Transporte informal, Modelos de elección binaria, Infracción de norma de transporte.

\section{DETERMINANTS ON DECISION AKING PROCESS OF MOTOCYCLE-TAXI DRIVERS IN CARTAGENA (COLOMBIA) TO VIOLATE TRANSIT REGULATION}

\begin{abstract}
Motorcycle-taxi is a driving informal public type of transportation which transit authorities have been trying to control in Cartagena city with regulations such as pico y placa. However, this regulation registers a high violation rate (about $69 \%$ of motorcycle-taxi drivers infringe it). In this paper, binary choice models are estimated in which examine factors that make a motorcycle-taxi drivers more likely to break the regulation. Results show that most robust determinants are the number of motorcycles use for labor purposes and the expected gain to get from it. It is concluded that the regulation has encouraged using of at least one additional motorcycle.

Keywords: Mototaxismo, Informal public transportation, Binary choice models, transport infringement.

Documento resultado del proyecto "Determinantes de la decisión de los mototaxistas en Cartagena (Colombia) de infringir la medida de pico y placa" cuyo objetivo es examinar los factores que hacen más propenso que un mototaxista infrinja la regulación de pico y placa. El proyecto pertenece Universidad de Cartagena.

* Magister en Economía y Magister en Economía del Medio Ambiente y de los Recursos Naturales, Profesora Asociada Programa de de Economía, Facultad de Ciencias Económicas de la Universidad de Cartagena. Miembro del Grupo de Investigación en Mercado Laboral. Correo electrónico: myanezc@unicartagena.edu.co.

*** Economista y Joven Investigadora del Grupo de Investigación de Mercado Laboral de la Facultad de Ciencias Económicas de la Universidad de Cartagena. Correo electrónico: kacevedog@unicartagena.edu.co.
\end{abstract}




\title{
DETERMINANTES NA DECISÃO DE MOTOTAXISTAS DE CARTAGENA (COLÔMBIA) DE INFRINGIR A MEDIDA DE RODÍZIO DE PLACA
}

\begin{abstract}
Resumo
O mototaxismo é um tipo de transporte público informal que se tentou controlar na cidade de Cartagena (Colômbia) com regulamentações como a de rodízio de número de placas. No entanto, esta regulação registra uma alta taxa de infração (aproximadamente 69\% dos mototaxistas não cumprem esta norma). Neste documento se trabalham modelos de escolha binária através dos quais se procuram examinar os fatores que fazem mais propenso a um mototaxista infringir a regulamentação. Os resultados mostram que os determinantes mais fortes são o número de motocicletas para trabalhar e o ganho esperado. Conclui-se que a medida incentivou o uso de pelo menos uma motocicleta adicional.
\end{abstract}

Palauras chave: Mototaxismo, Transporte informal, Modelos de escolha binária, Infração de norma de transporte.

Yánez, M. \& Acevedo, K. (2014) Determinantes de la decisión de los mototaxistas en Cartagena (Colombia) de infringir la medida de pico y placa. En: Revista de la Facultad de Ciencias Económicas de la Universidad Militar Nueva Granada. rev.fac.cienc.econ, XXII (1).

JEL: C35, D81, R41.

\section{Introducción}

El transporte de pasajeros en motocicletas, mototaxismo, es una actividad informal que tiene sus raíces en los profundos problemas sociales de las ciudades colombianas. Es así como la actividad que se inició en Cotorra (Córdoba) se extendió rápidamente en 26 de los 32 departamentos colombianos, convirtiéndose en una alternativa directa o indirecta de ingresos y de empleo para un sinnúmero de personas que no encuentran cabida en el sector formal.

La extensión y fortalecimiento del mototaxismo se han visto favorecidos por consideraciones que atañen tanto a la oferta como a la demanda del servicio. En particular, su rápida difusión obedece a un sistema de transporte público urbano ineficiente caracterizado por su insuficiente capacidad para absorber a la creciente demanda, los altos tiempos de viajes y la incomodidad. Además, el mototaxismo se presenta como una alternativa de bajo costo, de fácil acceso y como un servicio personalizado "puerta a puerta". No obstante, esta actividad genera externalidades negativas sobre el transporte público colectivo y la ciudadanía en general en cuanto presenta un mayor riesgo de accidentalidad y por su impacto en la contaminación atmosférica y auditiva.

En la ciudad de Cartagena, el fenómeno ha tomado dimensiones preocupantes. Según el Departamento Administrativo de Tránsito y Transporte de Cartagena (DATT), la autoridad local del transporte, el número de motocicletas que circulan en la ciudad pasó de 17.000 en el año 2004 a cerca de 65.000 en el 2012. Sólo entre los años 2011 y 2012 el crecimiento de estos vehículos fue de $38,3 \%$, comparado con el aumento en el total del parque automotor que fue de $21,1 \%^{1}$ y con el lento crecimiento en la malla vial ${ }^{2}$. Adicionalmente, entre 2008 y 2012 , las motocicletas pasaron de representar el $30 \%$ del parque automotor

Incluye motocicletas.

La infraestructura vial que se considera de buen estado pasó del 28\% en 2007 a 34\% en 2012. 
matriculado en el DAAT al 48,5\% (Cartagena Cómo vamos, 2012).

A pesar que las motocicletas representan menos del $50 \%$ del parque automotor y que son responsables de menos del $25 \%$ del número de accidentes de la ciudad, durante el 2012 estuvieron involucradas en el $68 \%$ de las muertes por accidentes y en el $74 \%$ de los heridos por esta causa (Proyecto Cartagena Cómo vamos, 2012). Por tanto, para controlar el fenómeno, las autoridades de tránsito han impuesto una serie de normatividades como la de pico y placa $^{3}$, y las restricciones a la circulación a determinadas horas, días y lugares. Sin embargo, estas normatividades no han controlado eficazmente el fenómeno debido al alto porcentaje de mototaxistas que infringen estas regulaciones.

Un estudio realizado por la Universidad de Cartagena (Yánez \& Villalba, 2009), demuestra que la infracción de la normatividad de pico en placa por parte de los mototaxistas es una práctica generalizada en la ciudad de Cartagena. Es así como el 57\% manifestó abiertamente haber infringido la normatividad por lo menos alguna vez, mientras que un $66 \%$ de los motociclistas fueron encuestados un día restringido para circular según la placa de la motocicleta.

Dado el alto porcentaje de infractores, esta investigación indaga por los factores que influyen en la decisión de infracción de la norma. Para esto, se estiman modelos de elección binaria que examinan los factores que hacen más propenso a un mototaxista a que infrinja la norma, destacando el papel de la ganancia esperada, la cual tiene importantes repercusiones de política por cuanto las autoridades tienen control sobre ella a través de las sanciones.

La estructura de esta investigación es la siguiente: en la segunda sección se examina el fenómeno del mototaxismo en Colombia y Cartagena visto desde una perspectiva histórica y social. En la tercera sección se expone la metodología de la estimación de los modelos de elección binaria de los determinantes de la probabilidad de que un mototaxista infrinja la medida; seguida de la caracterización de la población de análisis. En la quinta sección se presentan los resultados, y finalmente se concluye.

\section{El mototaxismo: un fenómeno multi-causal}

El sistema de transporte constituye un elemento crítico dentro de la estructura espacial de una ciudad debido a su importante papel en la decisión de localización de la población y de la actividad económica, y por esta vía, tiene importantes repercusiones en la competitividad, productividad y sostenibilidad ambiental de las ciudades. Lo anterior exige que la planificación del transporte se realice de manera continua y permanente en respuesta a una ciudad en permanente construcción.

En las ciudades colombianas ha surgido un tipo especial de transporte de pasajeros caracterizado por su informalidad. Este transporte que es conocido como mototaxismo, tiene sus raíces en los profundos problemas sociales de las ciudades colombianas, convirtiéndose a su vez en un problema social de enormes dimensiones. Asimismo, las deficiencias en el sistema de transporte público formal, la caída en el precio de las motocicletas y las facilidades de financiación han contribuido a la extensión y fortalecimiento de la problemática.

En Colombia, el transporte de pasajeros en motocicletas se originó alrededor de los años ochenta en el corregimiento de Cotorra (Córdoba) en respuesta a la necesidad de transporte de la población de este corregimiento hasta la carretera que conducía a Lorica o Montería. Los habitantes de Cotorra debían caminar aproximadamente tres kilómetros para lle-

\footnotetext{
Consiste en que de lunes a sábado los días pares sólo pueden circular en la ciudad las motocicletas que terminen en números pares (incluyendo el 0) y los días impares sólo pueden circular las motocicletas que terminen en números impares. Esta medida se implementó en el año 2005 con el decreto 0197 del Departamento Administrativo de Tránsito y Transporte de Cartagena (2005) con el objetivo de disminuir los índices de accidentalidad, reducir el flujo vehicular y mejorar la calidad ambiental de la ciudad. La medida de pico y placa también es aplicada a vehículos particulares y del servicio público (taxis), aunque con restricciones y características distintas.
} 
gar a la carretera los cuales eran casi imposibles de recorrer debido a la falta de vías. Dada esta necesidad, el mototaxismo fue visto como una alternativa de generación de ingresos para los desempleados de la región.

El mototaxismo creció rápidamente por las ciudades de la costa Caribe y posteriormente se extendió hacia casi todo el territorio nacional. Desde entonces, se ha convertido en la fuente de generación de ingresos de los sectores más desfavorecidos de las ciudades.

Varias investigaciones principalmente para las ciudades de las Costa Caribe han analizado las características socioeconómicas de la población que se dedica a esta actividad, las cuales encuentran que las personas jóvenes pertenecientes a los estratos más bajos y con niveles de educación secundaria constituyen el perfil promedio de esta población.

Para Cartagena, García \& González (2004) encuentran que cerca del $68,8 \%$ de los mototaxistas tienen entre 17 y 34 años de edad, el 72,04\% pertenece a los estratos 4 y 2 , y el $76,35 \%$ tiene educación secundaria (completa o incompleta). Estos resultados no distan en forma significativa de los encontrados por Yánez \& Villalba (2009) también para Cartagena, quienes resaltan que el $86 \%$ de la población mototaxista pertenece a los estratos 1 y 2 , y el $58 \%$ tiene entre 19 y 32 años de edad. Asimismo, el estudio El mototaxismo en Sincelejo, un análisis socioeconómico revela que el 90,7\% de los mototaxistas en dicha ciudad residen en estratos 1 y 2 , el $91,2 \%$ tiene edades entre los 14 y 43 años y el 50\% no alcanzó a terminar la secundaria.

Estas características socioeconómicas de la población mototaxista limitan sus posibilidades de encontrar empleos en el sector formal de la economía. De allí que no es de extrañar que la principal razón manifestada por los mototaxistas para dedicarse a la actividad es porque no encuentran otro trabajo ó por la falta de oportunidades de empleo (García \& Gon- zález, 2004; Yánez \& Villalba, 2009). A lo anterior, se suma el hecho de que el mototaxismo se presenta como una actividad rentable.

Al respecto, Sánchez (2011), quien analiza los factores que inciden en la probabilidad de ejercer el mototaxismo como principal actividad económica en Sincelejo, encuentra que la razón más importante es la mayor rentabilidad de esta actividad, por tanto, la recomendación del autor es que las normatividades que propendan por su reducción deben focalizarse en la disminución de los incentivos económicos de la actividad y no concentrarse en regulaciones prohibitivas. Ardila (2006) también sostiene que los decretos de prohibición del mototaxismo lo frenarán por un tiempo pero no lo eliminarán.

Si bien la falta de oportunidades constituye el caldo de cultivo para el surgimiento y avance de la actividad, existen otras razones relacionadas con la oferta y demanda del servicio que han incentivado el crecimiento desproporcionado del número de mototaxistas. Por el lado de la oferta, se destacan la disminución de los precios de las motocicletas como resultado de la reducción de los costos reales de la importación, las mayores facilidades de financiación y la inexistencia de barreras de entrada.

Por el lado de la demanda, se destaca la preferencia de los ciudadanos por este transporte, originado principalmente por la baja calidad del servicio de transporte público colectivo. Para el caso de Cartagena, el documento CONPES 3259, muestra que la demanda de transporte público de la ciudad creció entre los años 2002 y 2007 en un 6,8\%, el cual fue captado principalmente por el mototaxismo.

Asimismo, Segovia (2001) enumera los problemas específicos de este transporte en la ciudad, entre estos, la alta incomodidad en los vehículos (mal comportamiento de los conductores, mal estado de los vehículos, música ruidosa e inseguridad), percances en los desplazamientos (baja velocidad de desplazamiento, irregularidad en los tiempos de recorrido y

4 En Colombia existen 6 estratos socioeconómicos, clasificación que se establece según las características de los inmuebles residenciales. En los estratos 1 y 2 se encuentran las personas con menos recursos. 
ritmo débil de frecuencia) y disfuncionamiento en la estructura física de la red de transporte.

El documento CONPES 3620 reconoce el deterioro de la calidad del transporte público colectivo colombiano como se evidencia en velocidades promedios por debajo de los estándares en ciudades latinoamericanas con experiencias exitosas, altas edades del parque automotor y altos tiempos de desplazamiento.

Para el caso de Cartagena, el proyecto Cartagena Cómo Vamos estima que durante el primer semestre de 2009 los tiempos de desplazamiento oscilaron en 60 minutos (en un sólo sentido desde el Centro hacia la periferia o viceversa), con una velocidad promedio de desplazamiento de $16 \mathrm{Km} / \mathrm{h}$, niveles que se han mantenido estables desde el año 2005. Con respecto a las edades del parque automotor de la ciudad, los vehículos de transporte público urbano tienen 16 años de uso en promedio, asimismo el $47 \%$ y $31 \%$ de los buses y busetas, respectivamente, superaron los 20 años.

García \& González (2004) estimando modelos logísticos encuentran que el determinante más importante de la demanda del servicio de mototaxis en Cartagena es el menor tiempo de viaje. Además, resaltan que la elasticidad precio de la demanda es baja ya que los usuarios son relativamente insensibles a variaciones en el precio de la carrera, lo anterior a pesar que los usuarios están concentrados en estratos bajos. Con respecto a la elasticidad renta, los autores encuentran que la actividad se comporta como un servicio inferior: al aumentar la renta disminuye su demanda.

En esta misma línea, Toro, Alvis \& Arellano (2005) al estudiar los determinantes de la preferencia por cada uno de los tipos de transporte público, encuentran mediante la estimación de modelos probit que la variable más importante para explicar la demanda de mototaxis es la rapidez. La disminución en los tiempos de viaje permite a los ciudadanos destinarlo hacia actividades productivas.

Si bien el mototaxismo constituye una fuente importante de ingresos y empleos para numerosas familias, esta actividad genera riesgos y externalidades de consideración. El primer riesgo se relaciona con el mayor impacto de accidentalidad de este tipo de vehículos. Asimismo, su incremento desproporcionado, aumenta la contaminación atmosférica y auditiva, $e$ incrementa el consumo de combustibles fósiles que se encuentra como la causa principal del efecto invernadero y del calentamiento global.

\section{Datos y metodología}

La información socioeconómica de los mototaxistas en Cartagena (infractores y no infractores de la normatividad de pico y placa) fue obtenida de forma primaria mediante la aplicación de 288 encuestas durante los días 21 y 22 de mayo de 2009. El tamaño de muestra se obtuvo al aplicar la fórmula estadística para una población infinita, con un tamaño poblacional de 40.000 mototaxistas, un nivel de significancia del $5 \%$ y una probabilidad de éxito del 75\%, así:

$$
\mathrm{n}=\mathrm{Z}^{2} \frac{\mathrm{p} * \mathrm{q}}{\mathrm{i}^{2}}
$$

Donde:

$\mathrm{n}=$ Tamaño muestral.

$\mathrm{Z}=$ Valor correspondiente a la distribución de Gauss:

1,96 para un nivel de significancia de 0,05.

$\mathrm{p}=$ Prevalencia esperada del parámetro a evaluar.

$\mathrm{q}=(1-\mathrm{p})$

$\mathrm{i}=$ Error.

$\mathrm{N}=$ Tamaño de la población.

Estas encuestas fueron realizadas por estudiantes de la Universidad de Cartagena a mototaxistas que estuvieron dispuestos a participar en la encuesta y que se encontraban en alguna de las 249 estaciones de mototaxistas distribuidas por toda la ciudad. La identificación de estas estaciones se realizó mediante un trabajo de campo previo en el que se observaron los lugares donde se aglomeraban los mototaxistas para esperar pasajeros.

Para identificar a los mototaxistas infractores de la normatividad de pico y placa se adoptó el criterio de cotejar la placa y el día de realización de la encuesta ya que esta normatividad implica que las motocicletas con placas terminadas en un número par (impar) 
sólo pueden circular en la ciudad en un día par (impar). Además, se incluyeron aquellos mototaxistas que manifestaron infringir siempre o algunas veces la normatividad. Con este criterio se identificaron 200 infractores, quienes representan el 69,44\% de la población total.

\subsection{Determinantes de la infracción de la norma- tividad de pico y placa}

Debido a que existen ciertas características que hacen más probable que un mototaxista infrinja la normatividad de pico y placa, se estiman modelos de elección binaria (probit y logístico) para obtener las probabilidades de que un mototaxista sea infractor. Se tiene entonces que la variable latente no observable $y^{*}$, que determina el valor de la variable dependiente, equivalente a 1 cuando un mototaxista infringe la norma y 0 en caso contrario. Este valor viene dado por la función 2 .

$$
\begin{gathered}
y^{*}=X \beta+e \quad(2) \\
y^{*}\left\{1 \text { si } y^{*}>0 y 0\right. \text { en otro caso) }
\end{gathered}
$$

Bajo el supuesto que $y^{*}$ sigue una distribución normal estándar $(\varnothing)$, se tiene un modelo probit:

$$
\begin{aligned}
& P=\underset{y^{*}=\boldsymbol{X}_{i} \boldsymbol{\beta}}{E\left(\boldsymbol{X}_{i}^{*}\right)=\emptyset\left(\boldsymbol{X}_{i} \boldsymbol{\beta}\right)} \\
& =\frac{1}{\sqrt{2 \pi}} \int_{-\infty} e^{-Z^{2} / 2 d z}
\end{aligned}
$$

Al asumir que sigue una distribución logística $(\forall)$, se tiene un modelo logístico:

$$
\begin{gathered}
P=E\left(y^{*}=1 \mid \boldsymbol{X}_{i}\right)=\forall\left(\boldsymbol{X}_{i} \boldsymbol{\beta}\right) \\
=\frac{1}{1+e^{-\left(\boldsymbol{X}_{i} \boldsymbol{\beta}\right)}}
\end{gathered}
$$

Los parámetros $(\beta)$ son estimables por medio del método de máxima verosimilitud, como se aprecia en la ecuación 5 , donde $\mathrm{F}$ representa la distribución normal o logistica según sea el caso.

$$
\ln (L)=\sum_{i}\left\{y_{i} \ln \left[\mathrm{F}\left(\boldsymbol{X}_{i} \frac{\boldsymbol{\beta}}{\sigma}\right)\right]+\left(1-y_{1}\right) \ln \left[1-\mathrm{F}\left(\boldsymbol{X}_{i} \frac{\boldsymbol{\beta}}{\sigma}\right)\right]\right\}
$$

Los parámetros $(\beta)$ obtenidos deben ser ajustados para ser interpretados. Dado que tanto en el modelo probit como logístico $\mathrm{E}\left[\mathrm{y}^{*}\right]=\mathrm{F}\left(X_{i} \beta\right)$, los efectos marginales son: $\frac{\partial \mathrm{F}\left(\boldsymbol{X}_{\boldsymbol{i}} \boldsymbol{\beta}\right)}{\partial X_{i}}=\mathrm{F}\left(\beta X_{i}\right) \beta$.

\begin{tabular}{|c|c|}
\hline Variable & Indicador \\
\hline $\begin{array}{l}\text { Mototaxista infractor de la normativi- } \\
\text { dad de pico y placa }\end{array}$ & $1=$ Sí, $0=$ No \\
\hline Edad & Número de años cumplidos \\
\hline Estrato & $\begin{array}{l}\text { Estrato socioeconómico del ba- } \\
\text { rrio de residencia }\end{array}$ \\
\hline Experiencia & Número de años de mototaxista \\
\hline $\begin{array}{l}\text { Mototaxista con nivel de educación se- } \\
\text { cundaria (completa o incompleta) }\end{array}$ & $1=$ Sí, $0=$ No \\
\hline $\begin{array}{l}\text { Mototaxista con nivel de educación } \\
\text { técnica }\end{array}$ & $1=\mathrm{Sí}, 0=\mathrm{N}_{0}$ \\
\hline $\begin{array}{l}\text { Mototaxista con nivel de educación } \\
\text { universitaria }\end{array}$ & $1=\mathrm{Sí}, 0=$ No \\
\hline Personas a cargo & Número de personas a cargo \\
\hline Motos que maneja & Número de motos que maneja \\
\hline Cuenta bancaria & $1=\mathrm{SI} ; 0=$ No \\
\hline Otra actividad & $1=$ Sí; $0=$ No \\
\hline Ganancia esperada (primer quintil) & $1=$ Sí; $0=$ No \\
\hline
\end{tabular}

La tabla 1 muestra el conjunto de características observables (X) de los mototaxistas a fin de establecer su importancia en la probabilidad de infringir la normatividad de pico y placa.

Tabla 1. Operacionalización de las variables de los modelos de elección binaria ${ }^{5}$

La ganancia esperada se entiende como el valor medio de la distribución de probabilidad de los posibles resultados: ser sorprendido o no. Se calcula de la siguiente manera:

5 Fuente: Elaboración de las autoras. 
Ganancia esperada $=$ ganancia diaria $-p_{1}$ (valor del comparendo)

La ganancia diaria se obtiene de la diferencia entre los ingresos brutos, los costos directos (costo de la gasolina) y la tarifa para los no propietarios; p1 es la probabilidad de ser sorprendido por las autoridades infringiendo la normatividad de pico y placa, la cual se calcula como el cociente entre el número de mototaxistas sancionados por el Departamento de Tránsito y Transporte de Cartagena (DATT) por infracción de la normatividad y el número total de infractores en la ciudad durante los días 21 y 22 de mayo de 2009. La última información es obtenida de la expansión del número de infractores identificados en la muestra a la población total ( 40.000 mototaxistas). A continuación se presentan los resultados del cálculo de las probabilidades (tabla 2 y 3 ):

Tabla 2. Número total de infractores en Cartagena según día ${ }^{6}$

\begin{tabular}{|c|c|c|c|c|}
\hline Fecha & $\begin{array}{c}\text { Infractores } \\
\text { muestra }\end{array}$ & $\begin{array}{c}\text { Tamaño } \\
\text { muestra }\end{array}$ & $\begin{array}{c}\text { Porcentaje } \\
\text { infractores }\end{array}$ & $\begin{array}{c}\text { Infractores } \\
\text { población }\end{array}$ \\
\hline 21/05/2009 & 95 & 148 & 0,64 & 25.676 \\
22/05/2009 & 105 & 140 & 0,75 & 30.000 \\
\hline
\end{tabular}

Tabla 3. Probabilidad de ser sancionado $(p 1)^{7}$

\begin{tabular}{|c|c|c|c|}
\hline Fecha & Sancionados $^{*}$ & $\begin{array}{c}\text { Infractores } \\
\text { población }\end{array}$ & $\begin{array}{c}\text { Probabilidad } \\
\text { Sanción P(A) }\end{array}$ \\
\hline $21 / 05 / 2009$ & 25 & 25.676 & 0,000960 \\
22/05/2009 & 27 & 30.000 & 0,000887 \\
\hline
\end{tabular}

La sanción por infringir la norma incluye un comparendo de 15 salarios mínimos diarios vigentes, el cual durante el año 2009 ascendió a $\$ 248.450$. Los estadísticos de resumen de las variables incluidas en el modelo probit se aprecian en la tabla 4.
Tabla 4. Estadísticos de resumen ${ }^{8}$

\begin{tabular}{|c|c|c|c|c|c|}
\hline Variable** $^{* *}$ & 0bs & Mean & Std. Dev. & Min & Max \\
\hline Infractor (\%) & 288 & 0,69 & 0,46 & 0 & 1 \\
Edad & 288 & 32,23 & 8,09 & 19 & 62 \\
Estrato & 288 & 1,75 & 0,70 & 1 & 3 \\
Experiencia & 288 & 4,74 & 1,59 & 3 & 15 \\
$\begin{array}{c}\text { Educación secun- } \\
\text { daria (\%) }\end{array}$ & 288 & 0,72 & 0,45 & 0 & 1 \\
$\begin{array}{c}\text { Educación técnica } \\
\text { (\%) }\end{array}$ & 288 & 0,16 & 0,36 & 0 & 1 \\
$\begin{array}{c}\text { Educación universi- } \\
\text { taria (\%) }\end{array}$ & 288 & 0,03 & 0,17 & 0 & 1 \\
$\begin{array}{c}\text { Personas a cargo } \\
\text { Número de motos } \\
\text { Cuenta bancaria } \\
\text { (\%) }\end{array}$ & 288 & 28,65 & 1,50 & 0 & 9 \\
$\begin{array}{c}\text { Otra actividad (\%) } \\
\text { Ganancia esperada } \\
\text { (\%) }\end{array}$ & 288 & 0,36 & 0,48 & 1,00 & 2 \\
\hline
\end{tabular}

\section{Caracterización de la población mototaxista infractora y no infractora de la normatividad de pico y placa en Cartagena}

La infracción de la normatividad de pico en placa por parte de los mototaxistas es una práctica generalizada en Cartagena. Es así como 200 de los 288 encuestados, es decir, el 69,44\%, manifestó abiertamente infringir la normatividad por lo menos alguna vez y/o fueron encuestados un día restringido para circular en la ciudad (Ilustración 1).

El análisis de las características de los infractores muestra que las mayores tasas de infracción las registran los mototaxistas más jóvenes (en edades comprendidas entre los 19 y 39 años de edad) y que en general estas tasas decrecen a medida que aumenta

\footnotetext{
Fuente: cálculos de las autoras con base en información primaria y DATT.

Fuente: cálculos de las autoras con base en información primaria y DATT.

* Número promedio de sancionados en un día par e impar durante el año 2009.

8 Fuente: Elaboración de las autoras.

** El símbolo \% significa que las variables deben ser interpretadas en términos de porcentajes (multiplicar por 100).
} 
la edad. Si bien los mototaxistas con mayores de 53 años son más cautelosos en cuanto a la infracción de la norma, la tasa continúa siendo apreciable (50\%) (Ilustración 2).

Ilustración 1. Distribución de los infractores y no infractores, $2009^{9}$

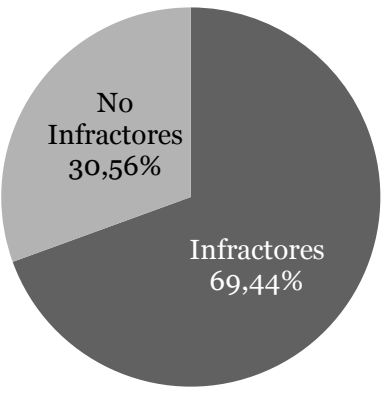

llustración 2. Tasa de infracción según rangos de edad, $2009^{10}$

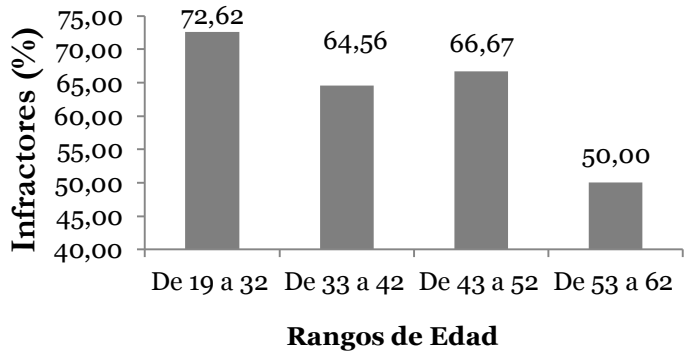

El estrato socioeconómico del mototaxista no parece mostrar una relación definida con las tasas de infracción, por lo que se deduce que el desacato de la medida de pico y placa es una práctica común en los mototaxistas independientemente de su estrato. En general, las tasas de infracción no fluctúan sustancialmente entre un estrato y otro $(69,3 \%$ en el estrato 1 y $68,4 \%$ en el estrato 3). Esta misma situación se observa si se examina el nivel educativo pues a diferencia de los mototaxistas con educación universitaria, las tasas de infracción no se ven alteradas sustancialmente por nivel educativo (Ilustración 3 y 4).

9 Fuente: Cálculos de las autoras con base en información primaria.

10 Fuente: Cálculos de las autoras con base en información primaria.

Fuente: Cálculos de las autoras con base en información primaria.

Fuente: Cálculos de las autoras con base en información primaria.

Fuente: Cálculos de las autoras con base en información primaria.
Ilustración 3. Tasa de infracción según estrato socioeconómico, 2009'11

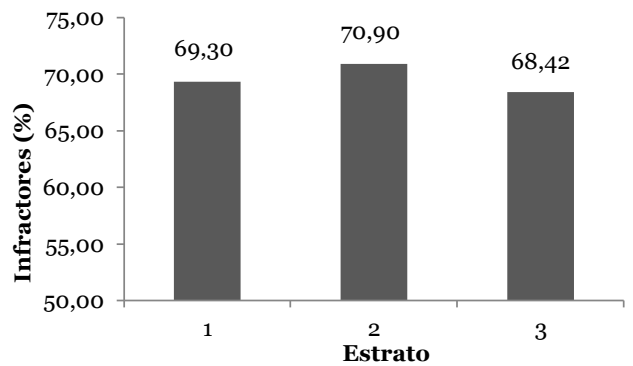

Ilustración 4. Tasa de infracción según nivel educativo, $2009^{12}$

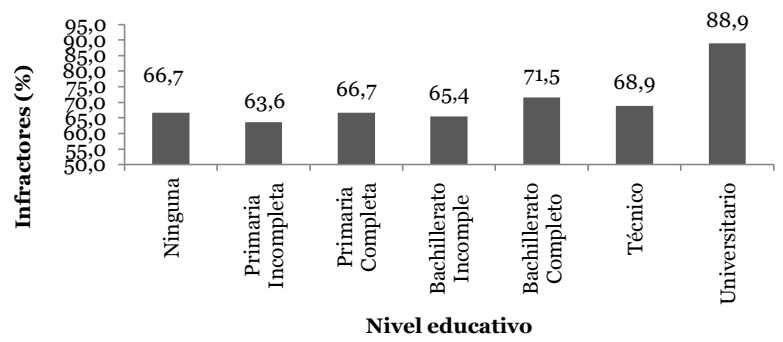

Por su parte, el número de años en la actividad que constituye una proxy de la experiencia muestra una relación directa con las tasas de infracción. Es así como todos los mototaxistas con más de 9 años ejerciendo la actividad infringieron la norma. Este hecho sugiere que la pericia en la actividad disminuye la percepción de riesgo asociada a ser sorprendidos (Ilustración 5).

llustración 5. Tasa de infracción según experiencia, $2009^{13}$

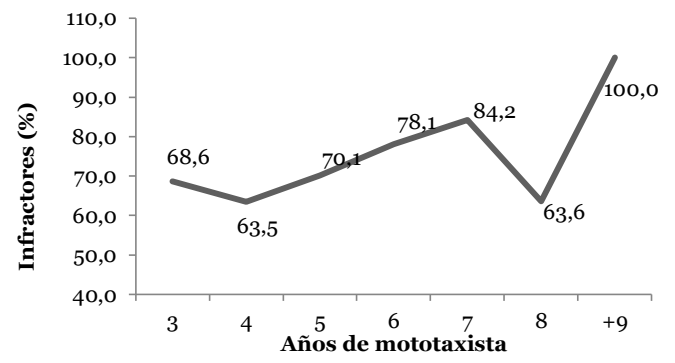


En cuanto al comportamiento de la tasa de infracción según el número de personas a cargo se observa una relación no lineal hasta las 6 personas dependientes del mototaxista, punto a partir del cual se reportan tasas de $100 \%$. Para capturar este efecto en los modelos de elección binaria se incluye el cuadrado de esta variable (Ilustración 6).

Ilustración 6. Tasa de infracción según número de personas a cargo, 2009'14

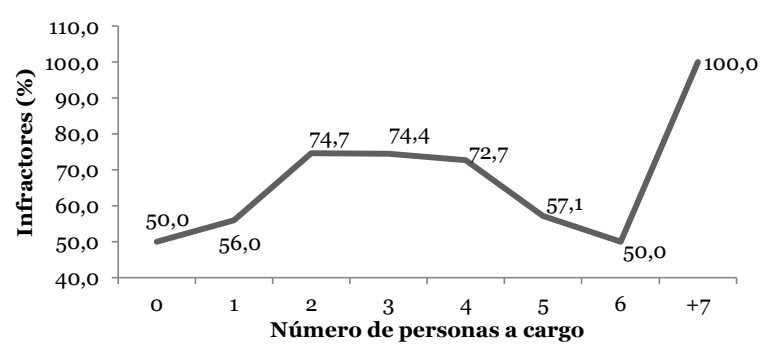

El uso de dos (2) motocicletas para ejercer la actividad constituye una salida de escape a la medida de pico y placa ya que quienes manejan dos motocicletas registran una tasa de infracción menor en cerca de 28,1 puntos porcentuales con respecto a aquellos que sólo manejan una (1). Este hecho tiene impactos económicos importantes al incentivar el mercado de las motocicletas y al desplazar al transporte público colectivo formal (Ilustración 7).

Ilustración 7. Tasa de infracción según número de motos que maneja, $2009^{15}$

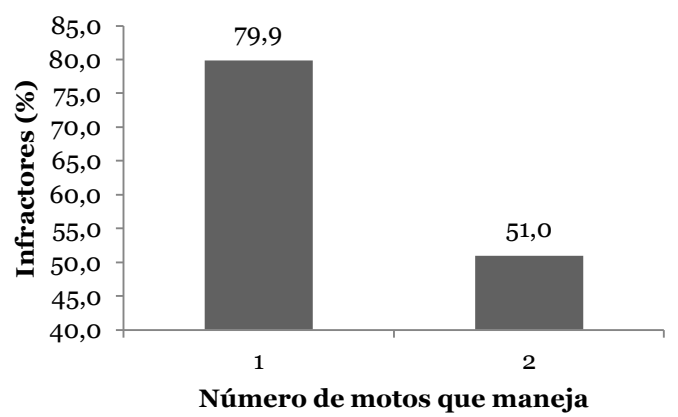

Fuente: Cálculos de las autoras con base en información primaria. Fuente: Cálculos de las autoras con base en información primaria. Fuente: Cálculos de las autoras con base en información primaria. Fuente: Cálculos de las autoras con base en información primaria.
Por su parte, el desarrollo de otra actividad económica no parece ser una salida de escape a la medida de pico y placa debido a que los mototaxistas que en forma paralela tienen otra fuente de ingresos reportan mayores tasas de infracción. Esto puede explicarse por el hecho que quienes obtienen recursos adicionales son más arriesgados a la hora de infringir la medida ya que cuentan con un respaldo adicional para enfrentar las sanciones. En general, las labores adicionales desarrolladas por estos son de carácter informal en actividades como la albañilería, ayudantes en busetas, mecánicos y electricistas (Ilustración 8).

Ilustración 8. Tasa de infracción según realización de otra actividad, $2009^{16}$

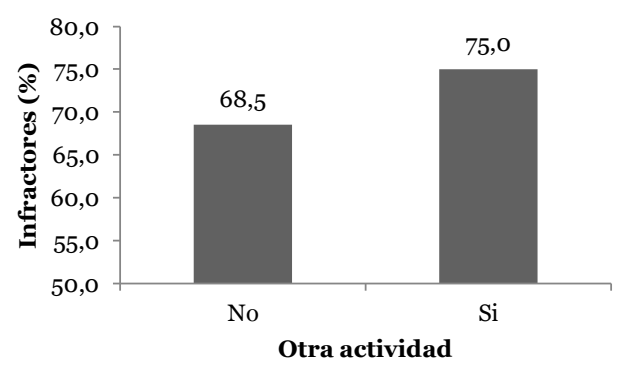

En cuanto a las tasas de infracción según tenencia de una cuenta bancaria, se encuentra que quienes poseen acceso al sistema bancario reportan mayores tasas de infracción, lo que puede atribuirse las mismas razones que explican las mayores tasas de quienes desarrollan otra actividad económica: cuentan con un respaldo adicional para enfrentar las sanciones (Ilustración 9).

Ilustración 9. Tasa de infracción según tenencia de cuenta bancaria, $2009^{17}$

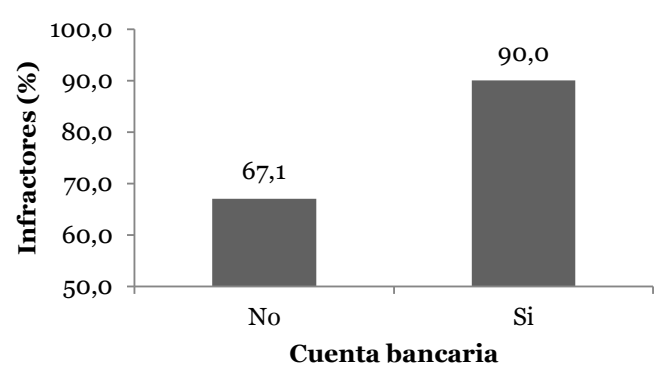


Finalmente, como es de esperar la ganancia esperada juega un papel importante en la decisión de desacato de la normatividad, de allí que se observe una relación directa entre los rangos de la ganancia esperada y las tasas de infracción (Ilustración 10 e Ilustración 11). Para los individuos en los que la ganancia esperada es un quintil más bajo, la tasa de infracción es inferior en aproximadamente 12 puntos porcentuales.

Ilustración 10. Tasa de infracción según ganancia esperada, $2009^{18}$

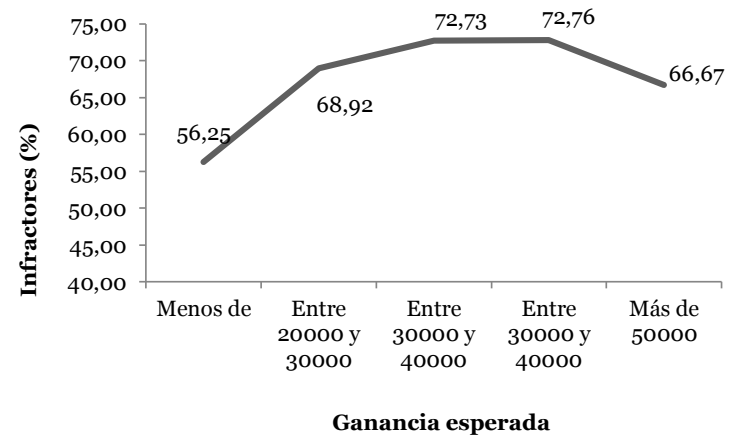

llustración 11. Tasa de infracción según quintil ganancia esperada, $2009^{19}$

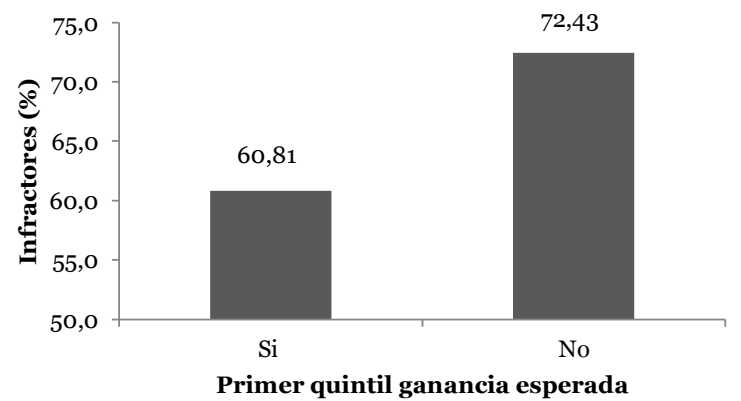

\section{Resultados de las estimaciones econométricas}

\subsection{Resultados de las estimaciones econométricas de la probabilidad de infringir la normatividad de pico y placa}

En primera instancia se estiman dos modelos de elección binaria: probit y logístico a fin evaluar cual modelo ajusta mejor el problema de estudio ${ }^{20}$. Los resultados de las estimaciones econométricas de la probabilidad que un mototaxista infrinja la normatividad de pico y placa se presentan en la tabla 5, la cual incluye coeficientes, errores estándares y niveles de significancia de cada una de las variables explicativas, y los estadísticos de contraste: razón de máxima verosimilitud (LR), estadístico de Wald con su probabilidad asociada ${ }^{21}$ y el pseudo $\mathrm{R}$ - cuadrado.

El análisis de la probabilidad del estadístico de Wald muestra que el conjunto de variables incluidas en el modelo explican la probabilidad de que un mototaxista sea infractor de la normatividad de pico y placa. Sin embargo, a nivel individual, las variables estrato socioeconómico del barrio de residencia, la educación y el desarrollo de otra actividad económica resultaron no significativas. Por su parte, el pseudo R- cuadrado se encuentra entre $16,4 \%$ y $16,7 \%$ el cual es relativamente bajo, aunque hay que tomar en cuenta que en los modelos de elección binaria la interpretación del $\mathrm{R}$ cuadrado no tiene sentido, por tanto, para valorar la bondad de ajuste del modelo se debe analizar la sensibilidad y especificidad.

En general, las variables explicativas presentan los signos esperados: la probabilidad que un mototaxista infrinja la normatividad tiene una relación directa con la experiencia y el número de personas a cargo, y una relación inversa con la edad y el número

\footnotetext{
Fuente: Cálculos de las autoras con base en información primaria.

Fuente: Cálculos de las autoras con base en información primaria.

Una primera especificación incluyo la variable "número de personas a cargo al cuadrado", la cual resultó no significativa.

21 Los modelos fueron estimados con errores estándares robustos por esta razón se obtiene el estadístico de Wald en lugar del estadístico de razón de máxima verosimilitud (LR).
} 
de motos para trabajar. Con respecto a las variables dicótomas, se encuentra que quienes tienen menos ganancia esperada (primer quintil) tienen menos probabilidades de infringir la norma, mientras que aquellos con acceso a los servicios bancarios tienen más probabilidades de desacatarla.

Tabla 5. Resultados de las estimaciones econométricas ${ }^{22}$

\begin{tabular}{|c|c|c|c|c|}
\hline \multirow{2}{*}{ VARIABLES } & \multicolumn{2}{|c|}{ PROBIT } & \multicolumn{2}{|c|}{ LOGIT } \\
\hline & Coeficiente & Error & Coeficiente & Error \\
\hline EDAD & $-0,025^{\star \star}$ & 0,012 & $-0,042^{* \star}$ & 0,019 \\
\hline EXPERIENCIA & $0,139^{* *}$ & 0,055 & $0,233^{* *}$ & 0,097 \\
\hline ESTRATO & $-0,050$ & 0,126 & $-0,099$ & 0,220 \\
\hline SECUNDARIA & 0,111 & 0,275 & 0,204 & 0,454 \\
\hline TECNICA & 0,097 & 0,351 & 0,168 & 0,594 \\
\hline UNIVERSITARIA & 0,581 & 0,699 & 1,439 & 1,421 \\
\hline PERSONAS A CARGO & $0,189^{*}$ & 0,068 & $0,315^{*}$ & 0,122 \\
\hline NMOTOS & $-0,952^{*}$ & 0,182 & $-1,631^{*}$ & 0,320 \\
\hline CUENTA & $0,936^{*}$ & 0,339 & $1,714^{\star *}$ & 0,685 \\
\hline OTRA ACTIVIDAD & $-0,125$ & 0,240 & $-0,185$ & 0,405 \\
\hline GANANCIA ESPERADA & $-0,571^{*}$ & 0,192 & $-1,025^{*}$ & 0,331 \\
\hline CONSTANTE & $1,603^{*}$ & 0,569 & $2,741^{*}$ & 0,971 \\
\hline Number of observations & \multicolumn{2}{|c|}{288} & \multicolumn{2}{|c|}{288} \\
\hline Log-Likelihood & \multicolumn{2}{|c|}{$-148,20$} & \multicolumn{2}{|c|}{$-147,75$} \\
\hline Wald chi2 & \multicolumn{2}{|c|}{44,677} & \multicolumn{2}{|c|}{38,785} \\
\hline Prob > chi2 & \multicolumn{2}{|c|}{0,000} & \multicolumn{2}{|c|}{0,000} \\
\hline Pseudo R2 & \multicolumn{2}{|c|}{0,164} & \multicolumn{2}{|c|}{0,167} \\
\hline note: ${ }^{*} p<0.01,{ }^{* *} p<0.05$ & 0.10 & & & \\
\hline
\end{tabular}

La no significancia de las variables de educación muestra que no hay diferencias estadísticamente significativas en la decisión de infringir la medida de pico y placa entre un mototaxista que tiene nivel educativo universitario, técnico o secundario con respecto a aquellos con educación primaria o ninguna (categoría de comparación). Estos resultados permiten sustentar la tesis que el desacato a la medida es independiente del nivel educativo del mototaxista. Esta misma conclusión aplica para el estrato socioeconómico del barrio de residencia y al desarrollo de otra actividad económica.
El análisis de los efectos marginales muestra que la variable más importante en el modelo econométrico es el número de motocicletas para ejercer la actividad ya que el aumento en una unidad disminuye la probabilidad de infringir la norma de pico y placa entre un $31,4 \%$ y $31,5 \%$. Este resultado sumado al hecho de que el número promedio de motocicletas por mototaxista fue de 1,36 en el año de aplicación de la encuesta y al crecimiento desbordado en los últimos años en el número de motocicletas que circulan por la ciudad ${ }^{23}$; sugieren que la normatividad de pico y placa incentivó el uso de por lo menos una motocicleta alternativa.

22 Fuente: Cálculo de las autoras.

23 Sólo entre los años 2011 y 2012 el crecimiento de estos vehículos fue de 38,3\% y entre 2008 y 2012 pasaron de representar el 30\% del parque automotor matriculado en el DAAT al $48,5 \%$. 
Tabla 6. Efectos marginales ${ }^{24}$

\begin{tabular}{|c|c|c|c|c|}
\hline \multirow{2}{*}{ VARIABLES } & \multicolumn{2}{|c|}{ PROBIT } & \multicolumn{2}{|c|}{ LOGIT } \\
\hline & Coeficiente & Efectos marginales & Coeficiente & Efectos marginales \\
\hline EDAD & $\begin{array}{c}-0,025^{\text {** }}(0,012) \\
(0,012)\end{array}$ & $\begin{array}{c}-0,008^{\star *} \\
(0,004)\end{array}$ & $\begin{array}{c}-0,042^{* *} \\
(0,019)\end{array}$ & $\begin{array}{c}-0,008^{* *} \\
(0,004)\end{array}$ \\
\hline EXPERIENCIA & $\begin{array}{l}0,139 * * \\
(0,055)\end{array}$ & $\begin{array}{l}0,046^{\star *} \\
(0,018)\end{array}$ & $\begin{array}{l}0,233^{* *} \\
(0,097)\end{array}$ & $\begin{array}{l}0,045^{\star *} \\
(0,019)\end{array}$ \\
\hline ESTRATO & $\begin{array}{l}-0,050 \\
(0,126)\end{array}$ & $\begin{array}{l}-0,016 \\
(0,042)\end{array}$ & $\begin{array}{l}-0,099 \\
(0,220)\end{array}$ & $\begin{array}{l}-0,019 \\
(0,042)\end{array}$ \\
\hline SECUNDARIA & $\begin{array}{c}0,111 \\
(0,275)\end{array}$ & $\begin{array}{c}0,037 \\
(0,093)\end{array}$ & $\begin{array}{c}0,204 \\
(0,454)\end{array}$ & $\begin{array}{c}0,040 \\
(0,091)\end{array}$ \\
\hline TECNICA & $\begin{array}{c}0,097 \\
(0,351)\end{array}$ & $\begin{array}{c}0,031 \\
(0,111)\end{array}$ & $\begin{array}{c}0,168 \\
(0,594)\end{array}$ & $\begin{array}{c}0,032 \\
(0,108)\end{array}$ \\
\hline UNIVERSITARIA & $\begin{array}{c}0,581 \\
(0,699)\end{array}$ & $\begin{array}{c}0,156 \\
(0,140)\end{array}$ & $\begin{array}{c}1,439 \\
(1,421)\end{array}$ & $\begin{array}{c}0,189 \\
(0,105)\end{array}$ \\
\hline PERSONAS CARGO & $\begin{array}{l}0,189^{\star} \\
(0,068)\end{array}$ & $\begin{array}{l}0,063^{*} \\
(0,023)\end{array}$ & $\begin{array}{l}0,315^{*} \\
(0,122)\end{array}$ & $\begin{array}{l}0,061^{*} \\
(0,024)\end{array}$ \\
\hline NMOTOS & $\begin{array}{l}-0,952^{*} \\
(0,182)\end{array}$ & $\begin{array}{l}-0,315^{\star} \\
(0,060)\end{array}$ & $\begin{array}{l}-1,631^{*} \\
(0,320)\end{array}$ & $\begin{array}{l}-0,314^{*} \\
(0,059)\end{array}$ \\
\hline CUENTA & $\begin{array}{l}0,936^{\star} \\
(0,339)\end{array}$ & $\begin{array}{l}0,230^{*} \\
(0,054)\end{array}$ & $\begin{array}{c}1,714^{* *} \\
(0,685)\end{array}$ & $\begin{array}{l}0,226^{* *} \\
(0,052)\end{array}$ \\
\hline OTRA ACTIVIDAD & $\begin{array}{l}-0,125 \\
(0,240)\end{array}$ & $\begin{array}{l}-0,042 \\
(0,084)\end{array}$ & $\begin{array}{l}-0,185 \\
(0,405)\end{array}$ & $\begin{array}{l}-0,037 \\
(0,083)\end{array}$ \\
\hline GANANCIA ESPERADA & $\begin{array}{l}-0,571^{\star} \\
(0,192)\end{array}$ & $\begin{array}{l}-0,201^{*} \\
(0,070)\end{array}$ & $\begin{array}{l}-1,025^{\star} \\
(0,331)\end{array}$ & $\begin{array}{l}-0,217^{\star} \\
(0,072)\end{array}$ \\
\hline CONSTANTE & $\begin{array}{l}1,603^{\star} \\
(0,569)\end{array}$ & & $\begin{array}{l}2,741^{*} \\
(0,971)\end{array}$ & \\
\hline Number of observations & 288 & & 288 & \\
\hline Log-Likelihood & $-148,20$ & & $-147,75$ & \\
\hline Wald chi2 & 44,677 & & 38,785 & \\
\hline Prob > chi2 & 0,000 & & 0,000 & \\
\hline Pseudo R2 & 0,164 & & 0,167 & \\
\hline
\end{tabular}

La ganancia esperada también resultó un importante determinante en la explicación de la decisión de infracción de la medida: quienes obtienen las menores ganancias esperadas tienen entre $20,1 \%$ y $20,7 \%$ menos probabilidad de desacatar la medida. No obstante, dado incentivo de los mototaxistas por hacer frente a la medida mediante el uso de una motocicleta alternativa y a las condiciones de mercado que facilitan la adquisición de una nueva motocicleta, se deduce que imponer sanciones más severas para disminuir la ganancia esperada de incumplir el pico y placa, lejos controlar el problema de accidentalidad, de movilidad y de contaminación asociados a estos vehículos, acrecentará el problema por el incremento en el número de motocicletas.

Entre otras variables, un año adicional en la edad disminuye la probabilidad de infringir la norma en un $0,8 \%$, lo que se explica porque los más jóvenes son menos adversos al riesgo; no obstante, un año

24 Fuente: Cálculo de las autoras. 
adicional de experiencia como mototaxista aumenta esta probabilidad de infracción entre $4,5 \%$ y $4,6 \%$, lo que puede atribuirse a que un año de experiencia no sólo aumenta la pericia de los mototaxistas en la actividad, sino en las estrategias para disminuir la probabilidad de sanción. Se destaca que el efecto de la experiencia es mayor al de la edad, contrarrestándola; por último, una persona adicional dependiente del mototaxista aumenta la probabilidad de infracción entre 6,1 y $6,3 \%$ y quienes tienen cuenta bancaria tienen un $23 \%$ más de probabilidad de infringir.

\section{Conclusiones}

La infracción de la normatividad de pico en placa por parte de los mototaxistas es una práctica generalizada en Cartagena debido a que cerca del $70 \%$ de manifestó abiertamente infringir la normatividad por lo menos alguna vez y/o fueron encuestados un día restringido para circular en la ciudad.

El análisis de las características tanto de la población mototaxista muestra que en general, las variables más importantes en la explicación del incumplimiento de la normatividad son las que repercuten en el cálculo de costos - beneficios de no cumplir la reglamentación y con las actitudes frente al riesgo. Es así como, la educación y el estrato fueron no significativas, pero sí la ganancia esperada, la edad, la experiencia y poseer una cuenta bancaria. También es significativa el número de personas a cargo.

No obstante, atacar factores como la ganancia esperada a través del aumento en la probabilidad de sanción o hacer más fuertes los castigos no son medidas efectivas para cumplir con los objetivos propuestos con la medida de pico y placa como el de reducir el impacto de accidentalidad y mejorar las condiciones ambientales de la ciudad, puesto que los mototaxistas están recurriendo a otra motocicleta para hacer frente a la restricción y poder así disponer de motiocicletas con placas par e impar.

\section{Referencias}

Ardila, A. (2006). "El mototaxismo: causas y posibles soluciones", Boletín informativo en línea [citado el 10 mayo del 2011]: Disponible en: http://www.voltairenet.org/article143679.html

Departamento Administrativo de Tránsito y Transporte de Cartagena (2005). Decreto 0197.

Documento Conpes 3259 (2003). Sistema integrado del servicio público urbano de transporte masivo de pasajeros del distrito de Cartagena - Transcaribe.

Documento Conpes 3620 (2009). Lineamientos de política para el desarrollo e impulso del comercio electrónico en Colombia. Disponible en: https:/www.dnp.gov.co/Portals/0/archivos/documentos/Subdireccion/Conpes/3620.pdf

García, I. \& González, P. (2004). "Características y determinantes del mercado del servicio de mototaxis en la ciudad de Cartagena". Trabajo de grado para optar por el título de economista, Universidad de Cartagena.

Ministerio de Transporte de Colombia (2006). Decreto 2961.

Proyecto Cartagena Cómo Vamos (2009). Cómo Vamos en Movilidad urbana: Tiempo y velocidad promedio del desplazamiento en transporte público en Cartagena. Disponible en: http://www. cartagenacomovamos.org/temp_downloads/Movilidad\%20 urbana\%20-\%20I\%20Semestre\%202009.pdf

Proyecto Cartagena Cómo Vamos (2012). Evaluación de la calidad de vida 2012. Disponible en: http://cartagenacomovamos.org/ temp_downloads/calidad-de-vida-2012.pdf

Sánchez, A. (2011). "La economía del mototaxismo: el caso de Sincelejo". Documento de trabajo de economía regional, No. 140.

Segovia, M. (2001). "Transporte público en Cartagena: una concentración de iniciativas individuales sin hilo conductor". Serie de estudios sobre la Costa Caribe, No. 16.

Toro, D., Alvis, J. \& Arellano, W. (2005). "Transporte público en Cartagena: ¿Qué factores determinan las preferencias de los usuarios?". En: Revista Economía y Región, 2(3).

Yánez, M. \& Villalba, J. (2009). "Evaluación socioeconómica de la normatividad del pico y placa sobre el mototaxismo en Cartagena". En: Revista Jornadas de investigación Facultad de Ciencias Económicas, 2(1):15 - 35. 
